\title{
Demographic factors do not predict weight loss maintenance in members of a commercial weight loss organisation
}

\author{
S. Whybrow ${ }^{1}$, A. McConnon ${ }^{2}$, M. Gibbs ${ }^{1}$, M. Raats ${ }^{2}$ and R. J. Stubbs ${ }^{3}$ \\ ${ }^{1}$ Food, Consumer Behaviour and Health Research Centre, Faculty of Health and Medical Sciences, University of Surrey, \\ Guildford GU2 7XH, ${ }^{2}$ Food, Consumer Behaviour and Health Research Centre, Department of Psychology, \\ University of Surrey, Guildford GU2 7XH and ${ }^{3}$ Slimming World, Alfreton DE55 4UE
}

\begin{abstract}
Demographic factors are important correlates of predisposition to obesity but much less is known about how they relate to weight loss and its maintenance. This analysis examined the demographic predictors of weight loss maintenance (WLM) in 1428 participants of a slimming organisation, who had been members for a mean \pm SD of $16 \pm 16$ months, had lost $13.8 \% \pm 9.2 \%$ weight and were trying to maintain, or increase, their weight loss during a subsequent 6 month study period.

Data were collected as part of the DiOGenes study ${ }^{(1)}$. Ethical approval was given by the University of Surrey Ethics Committee. Adults were recruited between August 2006 and July 2008 from Slimming World at group meetings and by email. Participants' weights (using calibrated scales) were taken from group records at four time points, measurement 1 (M1) at the start of the study period, nominally six months later (measurement 2 (M2)), six months before M1 and when they initially enrolled with Slimming World. Participants were free to continue following the weight-loss programme as they wished during this study, and there was no intervention other than completing the questionnaires. Participants completed a general screening questionnaire at M1 relating to age, gender, marital status, education level achieved, employment status, number of adults and children in the household, monetary expenditure on food, number of siblings, weight history, weight history of parents and siblings, medical history (whether a doctor had told them they have had obesity, diabetes, cancer, high blood pressure, high cholesterol, heart disease or stroke), medical history of parents and siblings, alcohol intake, smoking status, and birth weight. Linear regression analysis was used to identify the associations between questionnaire responses and weight change (as \% M1 weight) over the 6 month study period. Mean age was 46.8 years for women, 50.8 years for men; $95 \%$ were women.

There was no association between age, sex, marital status or family structure and subsequent WLM. Heavier people lost a greater percentage of their weight during the study period than did lighter people $(p<0.001)$, presumably because they had more to lose. People who were unemployed and "other" lost considerably more weight during the study than those who were employed, or not working for other reasons. However, there were only 37 and 58 people in these first two groups respectively. The percentage of respondents who reported being told by a doctor they had medical conditions was as follows: high blood pressure $30 \%$, high cholesterol $16 \%$ and obesity $36 \%$. Fewer than $5 \%$ of respondents answered yes for the other conditions. Being told by their GP that they were obese, either currently or in the past, significantly correlated with respondents' WLM $(p=0.007)$. All significant associations explained a very small percentage of the variance in WLM. No other demographic variables were associated with WLM. In this study demographic measures were used as baseline indicators of subsequent outcome. They did not change in the time course of the study. While demographic factors are likely to be important in predisposing people to weight gain and in accessing the means to lose weight, it is likely that individual changes in eating and activity behaviour are more important in determining successful weight loss and its maintenance.
\end{abstract}

This work was part of the Diet, Obesity and Genes project (www.diogenes-eu.org) funded by the European Commission (contract \#: Food-CT-2005513946) in the Food Quality and Safety Priority of the Sixth Framework Program.

1. Larsen TM \& Dalskov S et al. (2010) "The Diet, Obesity and Genes (Diogenes) Dietary Study in eight European countries - a comprehensive design for long-term intervention." Obesity Reviews 11, 76-91. 